\title{
Hair as a Biomarker of Long Term Mercury Exposure in Brazilian Amazon: A Systematic Review
}

\author{
Nathália Santos Serrão de Castro ${ }^{1, *(1)}$ and Marcelo de Oliveira Lima ${ }^{2}$ \\ 1 Centre of Research and Extension, Metropolitan College of Amazon (FAMAZ), Visconde de Souza \\ Franco Avenue, 72, Belém-Pará 66053-000, Brazil \\ 2 Environmental Section, Evandro Chagas Institute, BR-316, s/n, Ananindeua-Pará 67030-000, Brazil; \\ marcelolima@iec.pa.gov.br \\ * Correspondence: nathalia.serrao@famaz.edu.br
}

Received: 21 January 2018; Accepted: 28 February 2018; Published: 12 March 2018

\begin{abstract}
Many studies have assessed mercury $(\mathrm{Hg})$ exposure in the Amazonian population. This article performs a literature search of the studies that used hair as a biomarker of $\mathrm{Hg}$ exposure in the Brazilian Amazonian population. The search covered the period from 1996 to 2016 and included articles which matched the following criteria: (1) articles related to $\mathrm{Hg}$ exposure into Brazilian Amazon; (2) articles that used hair as a biomarker of Hg exposure; (3) articles that used analytical tools to measure the $\mathrm{Hg}$ content on hair and (4) articles that presented arithmetic mean and/or minimum and maximum values of $\mathrm{Hg}$. 36 studies were selected. The findings show that most of the studies were performed along margins of important rivers, such as Negro, Tapajós and Madeira. All the population presented mean levels of $\mathrm{Hg}$ on hair above $6 \mu \mathrm{g} \mathrm{g}^{-1}$ and general population, adults, not determined and men presented levels of $\mathrm{Hg}$ on hair above $10 \mu \mathrm{g} \mathrm{g}^{-1}$. The results show that most of the studies were performed by Brazilian institutions/researchers and the majority was performed in the State of Pará. The present study identified that Amazonian population has long-term been exposed to $\mathrm{Hg}$. In terms of future perspectives, this study suggests the implementation of a strategic plan for environmental health surveillance in the region in order to promote health and benefit Amazonian population.
\end{abstract}

Keywords: mercury; methylmercury; Amazon; hair Amazonia

\section{Background}

The health effects of methylmercury $(\mathrm{MeHg})$ exposure have been investigated since the accident that occurred in Minamata Bay, Japan. The clinical features of MeHg poisoning were classified as acute or chronic, based on the symptoms observed in patients living around the bay in the vicinity of the pollution source (a factory) and in patients living on the coast of the Shiranui Sea; both populations had consumed contaminated fish for almost 20 years [1].

Clinical studies of Japanese patients affected by dietary MeHg poisoning showed that mercury $(\mathrm{Hg})$ had long-term effects on health. The patients from Goshoura Island, an area close to Minamata Bay, who had mean levels of total hair $\mathrm{Hg}$ of $37 \mu \mathrm{g} \mathrm{g}^{-1}$ in $1960(n=16)$ and $2.4 \mu \mathrm{g} \mathrm{g}^{-1}$ in $2002(n=23)$ showed persistent sensory disorders caused by their past history of $\mathrm{MeHg}$ exposure [2,3]. Evaluation of data from a population-based study performed in 1971 at Goshoura showed an increased incidence of neurological signs, such as ataxia (12\%), dysarthria (5.9\%), and paresthesia of extremities (5.7\%) [4]. A study performed at Niigata, a town located along the Agano River that was affected by $\mathrm{MeHg}$ poisoning in 1965, showed that even people with chronic exposure to levels of $\mathrm{Hg}$ of less than $20 \mu \mathrm{g} \mathrm{g}^{-1}$ $(n=24)$ presented neurologic signs associated with $\mathrm{MeHg}$ poisoning [5]. Recently, a population-based study in Minamata and neighboring areas identified the association between neurological sign and the development of psychiatric symptoms. 
In spite of the findings observed after the Minamata disaster, there is no clear consensus yet about a dose-response relationship between $\mathrm{Hg}$ exposure and health effects given that the genetic characteristics of the populations vary, the modes and times of exposure are diverse, and that life-styles and behavior can influence on the toxic effects of $\mathrm{Hg}$ exposure [6,7]. However, vulnerable populations (i.e., pregnant women, human fetuses, neonates, and children) are under the potential risks of $\mathrm{Hg}$ effects [8-11].

Analysis of scalp hair has been a valuable method used to assess the $\mathrm{Hg}$ exposure of different populations because hair is easy to collect, store, and manipulate [12]. Hair Hg levels strongly correlate with an individual's dietary intake of $\mathrm{MeHg}$. Moreover, its chemical stability facilitates retrospective studies [13]. However, $\mathrm{Hg}$ can be incorporated into hair in other ways, such as the sorption of volatile species (i.e., elemental $\mathrm{Hg}$ ), and its level can be affected by hair color and growth rates, which are considered to be pre-analytical sources of variation that may cause bias and misleading interpretations $[14,15]$. In spite of these limitations, the versatility provided by scalp hair for assessing $\mathrm{Hg}$ exposure, especially in remote areas, has been valuable to access the $\mathrm{Hg}$ exposure in different populations.

In Brazil, many studies had been performed in the Amazonian region and hair has been selected as a biomarker of $\mathrm{Hg}$ exposure. The present article performs a systematic review of publications that analyzed $\mathrm{Hg}$ on hair of different populations into Brazilian Amazon. The objective of this article is to provide an overview of long-term exposure to $\mathrm{Hg}$ into Brazilian Amazon, identify populations under risk of $\mathrm{Hg}$ effects and give future perspectives for environmental health surveillance for the region.

\section{Methods}

This systematic review was registered in PROSPERO (registration number CRD42017056584). The search was performed using the following electronic databases: Pubmed, EBSCO, VHL (Virtual Health Library) and Scielo. Both authors performed independently the virtual search for articles titles and abstract using the search strategy showed in the Table 1 . The search covered the studies that were published in the period from 1996 to 2016. The present study followed the following inclusion criteria for articles: (1) articles related to $\mathrm{Hg}$ exposure into Brazilian Amazon; (2) articles that used hair as a biomarker of $\mathrm{Hg}$ exposure; (3) articles that used analytical tools to measure the $\mathrm{Hg}$ content on hair and (4) articles that presented arithmetic mean and/or minimum and maximum values of $\mathrm{Hg}$. As exclusion criteria, the present study followed: (1) review articles of $\mathrm{Hg}$ exposure and (2) articles which methodology was not clear, such as sample size, locality and analytical tools. The potential studies were screened and duplicates were removed. Articles not excluded were read by the two authors who extracted in detail the geographic location where the study was performed, sample size and type, the age range and/or mean age of the populations, the first author of the study, the year of the publication, and the arithmetic mean of $\mathrm{Hg}$ observed.

Table 1. Search strategies in electronic database.

\begin{tabular}{cc}
\hline Strategy & Keywords \\
\hline$\# 1$ & Mercury and hair and Amazon \\
$\# 2$ & Methylmercury and hair and Amazon \\
$\# 3$ & Mercury and Brazil and Amazonia \\
$\# 4$ & Mercury and Amazon \\
\hline
\end{tabular}

The present study classified the type of the studied populations as follows: general population ( 0 to $>60$ years old), adult population ( $>15$ years old), children $(<15$ years old), women, men, indigenous and not determined (nd) when the study did not identify the type and/or the age range of the target population. 
According to the type of each population, the data of the mean level of $\mathrm{Hg}$ was used in order to determine a weighted average in which the number of individuals studied contributed equally to a final average (Equation (1)).

$$
W A=((X 1 \times I 1)+(X 2 \times I 2)+(X n \times I n)) /(\Sigma I 1+I 2+I n)
$$

where: $W A=$ weighted average; $X=$ arithmetic mean of $\mathrm{Hg}\left(\mu \mathrm{g} \mathrm{g}^{-1}\right) ; I=$ Number of Individuals.

A cartographic quali-quantitative analysis of the data was performed. The classification of the populations determined for this study were considered for a qualitative evaluation and a quantitative analysis based on the degree of exposure followed the following criteria: lower (less than $2 \mu \mathrm{g} \mathrm{g}^{-1}$ ), medium (between 2 and $6 \mu \mathrm{g} \mathrm{g}^{-1}$ ) and higher (above $6 \mu \mathrm{g} \mathrm{g}^{-1}$ ).

The ArcMap 10.1 software (ESRI, Redlands, CA, USA) was used for georeferencing for studied where the geographic coordinates were not available. The geographic location was matched with the Brazilian Institute of Geography and Statistics (IBGE). The quantitative analysis was evaluated by a "choropleth map" where the differences of $\mathrm{Hg}$ exposure according to different populations and regions were shaded: yellow representing populations with low exposure to $\mathrm{Hg}$ and red high exposure.

Among the major source of risk of bias of the present study we can cite here:

(1) The period of the study.

The present study searched for studies that were published from 1996 to 2016 (20 years). Thus, the concentration of $\mathrm{Hg}$ on hair among the populations can vary, and, thus, the general visualization provided by the cartographic quali-quantitative analysis could not represent the current status.

(2) The methodologies for $\mathrm{Hg}$ measurement were not an exclusion or inclusion criteria.

Once the present study considered valid all the methodologies that measured $\mathrm{Hg}$ on hair, the results can not reflect a "gold standard" for $\mathrm{Hg}$ exposure in Amazon. Instead, all the selected articles were peer reviewed, thus the data could be considered to give an overview of long term $\mathrm{Hg}$ exposure in Amazonian population.

\section{Results}

The database search identified 1283 articles, of which 973 were duplicated and, consequently, removed. The potential 310 articles were screened and 274 of them were removed given that did not attended to some inclusion/exclusion criteria: 169 used other matrices rather than hair, 42 which methodology was not clear, 27 were review articles, 27 were performed in other regions rather than Brazilian Amazon (7 in Peru, 6 in Bolivia, 4 in Ecuador, 3 in French Guiana, 1 in Venezuela, 1 in Suriname, 1 in Peru and Ecuador, 1 Faroe Island, 1 in Colombia, 1 in Africa and 1 in the Northwest of Brazil), 5 were published out of the period of investigation and 4 contained generalist subject about $\mathrm{Hg}$ exposure. The study selection is summarized in the flowchart described in Figure 1.

In this context, the present study comprised 36 articles, comprising a total of 11,827 individuals (Table 2). According to the year of publication, the articles were published as follows: three in 1998 [16-18], two in 1999 [19,20], three in 2000 [21-23], two in 2001 [24,25], one in 2002 [26], two in 2003 [27,28], four in 2005 [29-32], three in 2006 [33-35], two in 2007 [36,37], one in 2008 [38], one in 2009 [39], two in 2010 [40,41], three in 2012 [42-44], one in 2013 [45], one in 2014 [46], three in 2015 [47-49] and two in 2016 [50,51]. Most of the studies (61\%) were performed exclusively by Brazilians Institution $[16,18,20-22,24,28-34,37,41-46,49,51]$ and others $(36 \%)$ had been performed in collaboration with them $[17,19,23,25,26,35,36,38-40,47,48,50]$. Only one study $(3 \%)$ was developed for a foreign country solely [27]. 


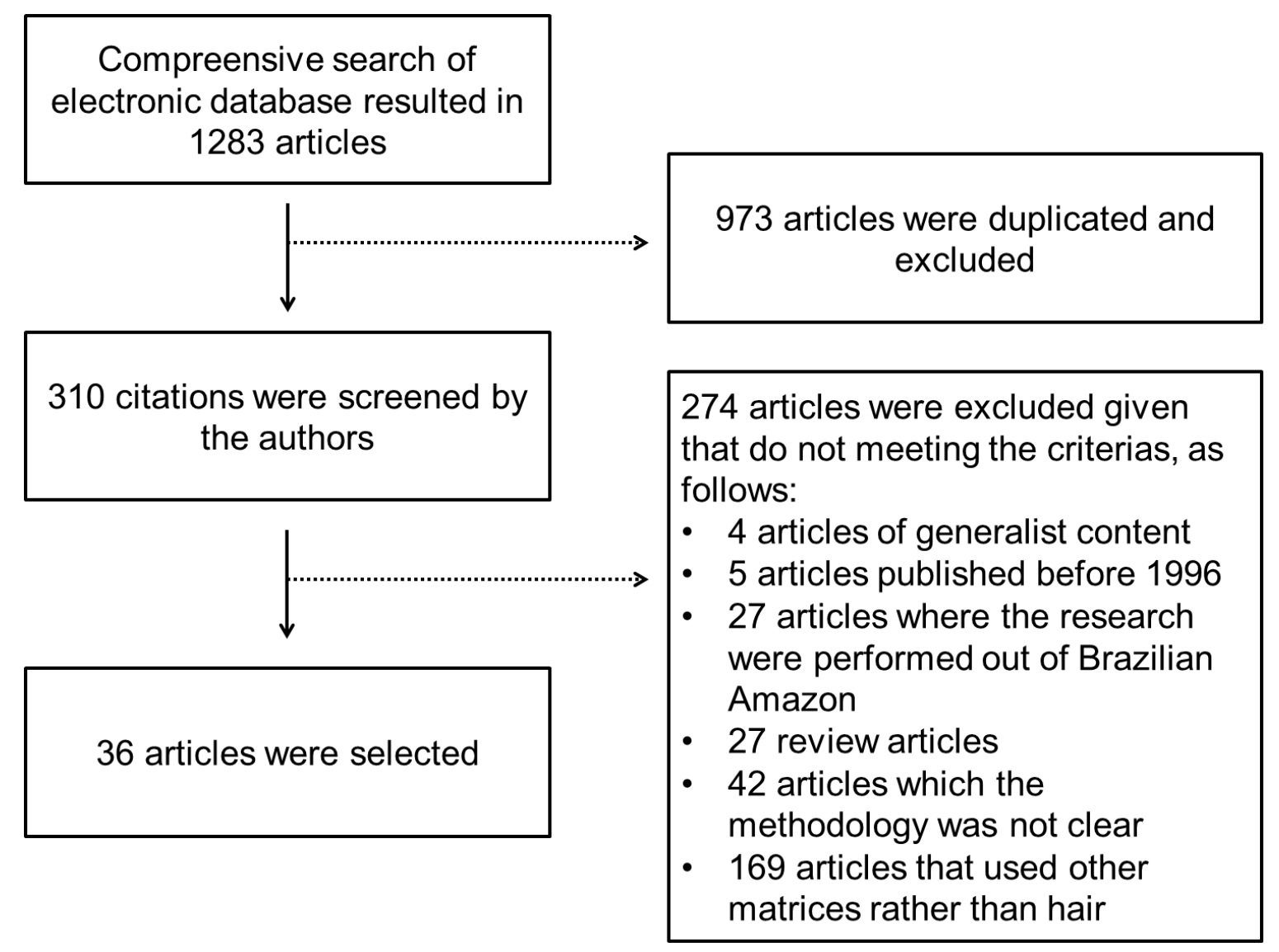

Figure 1. Study selection flowchart. 
Table 2. Characteristics of the included studies $(n=36)$.

\begin{tabular}{|c|c|c|c|c|c|c|c|c|c|}
\hline Year & Author & Locality & State & $n$ & $\begin{array}{l}\text { Type of Population According to } \\
\text { the Author }\end{array}$ & Population Specificity & $\begin{array}{l}\text { Type of the Population According } \\
\text { to the Present Study }\end{array}$ & Range $\mathrm{Hg}\left(\mu \mathrm{g} \mathrm{g}^{-1}\right)$ & Mean $\mathrm{Hg}\left(\mu \mathrm{g} \mathrm{g}^{-1}\right)$ \\
\hline \multirow{4}{*}{1998} & \multirow{4}{*}{ Barbosa A.C. [16] } & Fresco River & PA & 28 & Kayapo women & childbearing women & I & $0.8-13.7$ & 8.11 \\
\hline & & Madeira River & RO & 98 & non-indigenous women & childbearing women & $\mathrm{W}$ & $2.6-94.7$ & 14.08 \\
\hline & & Fresco River & PA & 54 & Kayapo children & nd & I & $2.0-20.4$ & 7.30 \\
\hline & & Madeira River & & 71 & non-indigenous children & nd & $\mathrm{C}$ & $0.8-44.4$ & 10.82 \\
\hline \multirow{5}{*}{1998} & \multirow{5}{*}{ Kehrig H.A. [17] } & \multirow{5}{*}{ Balbina Village } & \multirow{5}{*}{ AM } & 53 & total of population studied & nd & G & nd & 6.54 \\
\hline & & & & 16 & children & female & $\mathrm{C}$ & $1.3-22.0$ & 7.7 \\
\hline & & & & 12 & children & male & C & 2.5-11.4 & 5.3 \\
\hline & & & & 12 & adults & female & A & $2.2-15.5$ & 7.4 \\
\hline & & & & 13 & adults & male & A & $1.2-12.2$ & 5.5 \\
\hline \multirow[b]{2}{*}{1998} & \multirow{2}{*}{ Barbosa A.C. [18] } & \multirow{2}{*}{ Madeira River } & \multirow[b]{2}{*}{ RO } & 37 & women & nd & $\mathrm{W}$ & $2.0-37.2$ & 14.3 \\
\hline & & & & 37 & children & $0.5-15$ months & C & $1.4-34.2$ & 9.8 \\
\hline \multirow{10}{*}{1999} & \multirow{10}{*}{$\begin{array}{l}\text { Silva-Forsberg } \\
\text { M.C. [19] }\end{array}$} & total (all the populations studied) & \multirow{10}{*}{$\mathrm{AM}$} & 154 & total of population studied & $0.2-66$ y.o & G & $5.76-171.24$ & 75.46 \\
\hline & & Acariquara, Rio Urubaxi & & 15 & nd & $0.3-56$ y.o & G & $14.37-146.25$ & 69.18 \\
\hline & & Tupuruquara, Rio Marie & & 57 & nd & $0.2-66$ y.o & G & $10.44-171.24$ & 97.44 \\
\hline & & Macuna, Rio Uneiuxi & & 17 & nd & $0.7-52$ y.o & G & $22.17-129.19$ & 76.75 \\
\hline & & Perseverança, Rio Negro & & 23 & nd & $0.2-65$ y.o & G & $15.77-122.32$ & 65.72 \\
\hline & & Ilha do Pinto. Rio Negro & & 12 & nd & $1.6-37$ y.o & G & $19.02-100.95$ & 69.58 \\
\hline & & Tapera. Rio Padauari & & 11 & nd & $2-59$ y.o & G & $19.20-55.59$ & 37.48 \\
\hline & & Tapereira. Rio Negro & & 10 & nd & $2-47$ у.о & G & $24.94-110.51$ & 69.10 \\
\hline & & Aldeia Maia. Rio Maia & & 7 & nd & $13-40$ у.о & G & $5.76-63.02$ & 28.02 \\
\hline & & Sitio Velho. Rio Marauia & & 2 & nd & $42-62$ y.o & A & $13.93-62.57$ & 38.25 \\
\hline \multirow{2}{*}{1999} & Guimaraes & Pracuuba Lake & \multirow[b]{2}{*}{$\mathrm{AP}$} & 15 & fishermen and their family & nd & nd & nd & 16.7 \\
\hline & J.R.D. [20] & Duas Bocas Lake & & 15 & fishermen and their family & nd & nd & nd & 28 \\
\hline 2000 & Hacon S. [21] & Alta Floresta & MT & 75 & pregnant women & $14-45$ y.o & $\mathrm{W}$ & $0.051-8.2$ & 1.12 \\
\hline \multirow{26}{*}{2000} & \multirow{26}{*}{ Santos E.C.O. [22] } & & \multirow{26}{*}{$\mathrm{PA}$} & 220 & total of population studied & $0 \rightarrow 65$ у.о & G & $0.53-49.99$ & 11.75 \\
\hline & & & & 30 & nd & $0-5$ у.о & C & 1.09-20.46 & 5.84 \\
\hline & & & & 68 & nd & $6-10$ y.o & C & $0.70-35.80$ & 13.06 \\
\hline & & & & 33 & nd & $11-15$ у.о & C & $1.22-47.00$ & 14.2 \\
\hline & & & & 12 & nd & $16-20$ y.o & A & $5.56-19.90$ & 13.39 \\
\hline & & & & 10 & nd & $21-25$ y.o & A & $1.40-29.50$ & 15.25 \\
\hline & & & & 9 & nd & $26-30$ y.o & A & $3.70-21.40$ & 11.06 \\
\hline & & Brasília Legal & & 16 & nd & $31-35$ у.о & A & $2.84-37.20$ & 12.57 \\
\hline & & & & 12 & nd & $36-40$ у.о & A & $5.0-33.0$ & 14.21 \\
\hline & & & & 1 & nd & $41-45$ у.о & A & nd & 11.7 \\
\hline & & & & 7 & nd & $46-50$ у.о & A & $1.02-14.24$ & 7.06 \\
\hline & & & & 8 & nd & $51-55$ у.о & A & $3.57-49.99$ & 11.53 \\
\hline & & & & 5 & nd & $56-60$ y.o & A & $0.53-7.07$ & 4.93 \\
\hline & & & & 6 & nd & $61-65$ у.о & A & $5.01-15.94$ & 11.33 \\
\hline & & & & 3 & nd & $>65$ y.o & A & $2.78-16.46$ & 7.45 \\
\hline & & \multirow{11}{*}{ São Luiz do Tapajós } & & 327 & total of population studied & $0 \rightarrow 65$ y.o & G & $0.10-94.50$ & 19.91 \\
\hline & & & & 75 & & $0-5$ у.о & C & $0.10-94.50$ & 21.06 \\
\hline & & & & 74 & nd & $6-10$ у.о & C & $2.40-52.50$ & 22.1 \\
\hline & & & & 51 & nd & $11-15$ y.o & C & $3.90-61.80$ & 23.24 \\
\hline & & & & 21 & nd & $16-20$ y.o & A & $2.10-33.60$ & 19.11 \\
\hline & & & & 21 & nd & $21-25$ у.о & A & $1.73-32.0$ & 15.68 \\
\hline & & & & 15 & nd & $26-30$ y.o & A & $3.90-34.90$ & 15.34 \\
\hline & & & & 15 & nd & $31-35$ у.о & A & $5.10-38.0$ & 18.98 \\
\hline & & & & 15 & nd & $36-40$ y.o & A & $2.60-27.8$ & 14.31 \\
\hline & & & & 14 & nd & $41-45$ y.o & A & $3.20-33.60$ & 15.13 \\
\hline & & & & 8 & nd & $46-50$ y.o & A & $4.0-47.0$ & 21.71 \\
\hline
\end{tabular}


Table 2. Cont.

\begin{tabular}{|c|c|c|c|c|c|c|c|c|c|}
\hline Year & Author & Locality & State & $n$ & $\begin{array}{l}\text { Type of Population According to } \\
\text { the Author }\end{array}$ & Population Specificity & $\begin{array}{l}\text { Type of the Population According } \\
\text { to the Present Study }\end{array}$ & Range $\mathrm{Hg}\left(\mu \mathrm{g} \mathrm{g}^{-1}\right)$ & Mean $\mathrm{Hg}\left(\mu \mathrm{g} \mathrm{g}^{-1}\right)$ \\
\hline \multirow{18}{*}{2000} & \multirow{18}{*}{ Santos E.C.O. [22] } & \multirow{4}{*}{ São Luiz do Tapajós } & \multirow{18}{*}{ PA } & 4 & nd & $51-55$ у.о & $\mathrm{A}$ & $5.90-20.60$ & 15.4 \\
\hline & & & & 6 & nd & $56-60$ y.o & A & $7.90-27.60$ & 17.13 \\
\hline & & & & 3 & nd & $61-65$ у.о & A & $3.8-27.80$ & 12.6 \\
\hline & & & & & nd & $>65$ y.o & A & $3.20-20.80$ & 12.6 \\
\hline & & \multirow{14}{*}{ Santana de Ituqui } & & 321 & total of population studied & $0->65$ у.о & G & $0.40-11.60$ & 4.33 \\
\hline & & & & 37 & nd & $0-5$ у.о & C & $0.50-8.50$ & 3.67 \\
\hline & & & & 81 & nd & $6-10$ у.о & C & $0.40-10.9$ & 4.44 \\
\hline & & & & 62 & nd & $11-15$ y.o & C & $2.0-11.6$ & 4.47 \\
\hline & & & & 25 & nd & $16-20$ y.o & A & $2.5-9.60$ & 5 \\
\hline & & & & 17 & nd & $21-25$ y.o & A & $1.30-7.10$ & 3.34 \\
\hline & & & & 16 & nd & $26-30$ y.o & A & $1.70-9.20$ & 4.69 \\
\hline & & & & 19 & nd & $31-35$ у.о & A & $1.90-9.60$ & 5.36 \\
\hline & & & & 18 & nd & $36-40$ у.о & A & $1.20-6.0$ & 3.44 \\
\hline & & & & 10 & nd & $41-45$ у.о & A & $2.70-6.80$ & 4.18 \\
\hline & & & & 10 & nd & $46-50$ y.o & A & $1.90-6.40$ & 4.02 \\
\hline & & & & 9 & nd & $51-55$ у.о & A & $2.30-9.0$ & 5.39 \\
\hline & & & & 6 & nd & $56-60$ y.o & A & $3.10-6.90$ & 4.37 \\
\hline & & & & 4 & $\begin{array}{l}\text { nd } \\
\text { nd }\end{array}$ & $61-65$ y.o & A & $\begin{array}{l}1.90-9.0 \\
070-570\end{array}$ & 4.15 \\
\hline 2000 & Dolbec J. [23] & Cametá & PA & 68 & total of population studied & $12-79$ у.о & G & nd & 10.8 \\
\hline \multirow{2}{*}{2001} & \multirow{2}{*}{ Barbosa A.C. [24] } & \multirow{2}{*}{ Negro River } & \multirow{2}{*}{$\mathrm{AM}$} & 73 & children & $<15$ y.o & C & $0.51-45.89$ & 18.52 \\
\hline & & & & 76 & adults & $>15$ y.o & A & $1.66-59.01$ & 21.4 \\
\hline \multirow{4}{*}{2001} & \multirow{4}{*}{ Harada M. [25] } & Barreiras & \multirow{4}{*}{ PA } & 76 & fisherman and family & $1-67$, mean 28 y.o & G & $1.8-53.8$ & 16.4 \\
\hline & & Rainhas & & 12 & fisherman and family & $7-53$, mean 31 y.o & G & $3.1-34.5$ & 14.1 \\
\hline & & São Luiz do Tapajós & & 44 & fisherman and family & $3-47$, mean 21 y.o & G & $5.1-42.2$ & 20.8 \\
\hline & & $\begin{array}{l}\text { Special group from Barreiras, Rainha and } \\
\text { São Luiz do Tapajós }\end{array}$ & & 50 & $\begin{array}{l}\text { eligible subjects examined } \\
\text { clinically that presented high level } \\
\text { of } \mathrm{Hg}(>20 \mathrm{ppm}) \text { from March } 1994 \\
\text { to February } 1998\end{array}$ & 3-65, mean 25 y.o & G & $5.1-42.7$ & 23.6 \\
\hline \multirow{3}{*}{2002} & \multirow{3}{*}{ Crompton P. [26] } & \multirow{3}{*}{ Jacareacanga } & \multirow{3}{*}{ PA } & 205 & total of population studied & $\begin{array}{l}\text { general population, } \\
\text { except children under }\end{array}$ & G & $0.3-83.2$ & 8.6 \\
\hline & & & & nd & 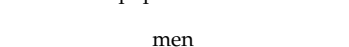 & $\begin{array}{l}2 \text { y.o } \\
\text { nd }\end{array}$ & $\mathrm{M}$ & nd & 11.0 \\
\hline & & & & nd & women & nd & $\mathrm{W}$ & nd & 6.7 \\
\hline 2003 & Passos C.J. [27] & Brasília Legal & PA & 26 & adults women & $23-62$, mean 41 y.o & $\mathrm{W}$ & $4.0-20.0$ & 10.0 \\
\hline \multirow{9}{*}{2003} & \multirow{9}{*}{ Santos E.C.O. [28] } & \multirow{9}{*}{ Guajará Mirim e Nova Mamoré } & \multirow{9}{*}{ RO } & 910 & total of studied population & $0->45$ y.o & I & $0.52-83.89$ & 8.37 \\
\hline & & & & 57 & nd & $0-2$ у.о & I & $1.48-83.89$ & 10.54 \\
\hline & & & & 115 & nd & $3-5$ y.o & I & $1.67-47.22$ & 9.34 \\
\hline & & & & 152 & nd & $6-10$ y.o & I & $0.52-63.81$ & 8.16 \\
\hline & & & & 114 & nd & $11-15$ y.o & I & $0.65-31.11$ & 6.86 \\
\hline & & & & 177 & nd & $16-25$ y.o & I & $0.65-39.42$ & 8.45 \\
\hline & & & & 114 & nd & $26-35$ y.o & I & $1.37-28.64$ & 8.56 \\
\hline & & & & 50 & nd & $36-45$ y.o & I & $1.49-21.25$ & 8.39 \\
\hline & & & & 131 & nd & $>45$ y.o & I & $1.37-25.84$ & 7.84 \\
\hline \multirow[t]{2}{*}{2005} & \multirow[t]{2}{*}{ Dorea J.G. [29] } & \multirow[t]{2}{*}{ Teles Pires (Tapajós Basin) } & \multirow[t]{2}{*}{ PA } & 47 & Kayabi indigenous & $\begin{array}{l}\text { Kayabi community } \\
\text { from Teles Pires }\end{array}$ & I & nd & 12.8 \\
\hline & & & & 249 & Munduruku indigenous & $\begin{array}{l}\text { Munduruku community } \\
\text { from Tales Pires }\end{array}$ & I & nd & 3.4 \\
\hline
\end{tabular}


Table 2. Cont

\begin{tabular}{|c|c|c|c|c|c|c|c|c|c|}
\hline Year & Author & Locality & State & $n$ & $\begin{array}{l}\text { Type of Population According to } \\
\text { the Author }\end{array}$ & Population Specificity & $\begin{array}{l}\text { Type of the Population According } \\
\text { to the Present Study }\end{array}$ & Range $\mathrm{Hg}\left(\mu \mathrm{g} \mathrm{g}^{-1}\right)$ & Mean $\mathrm{Hg}\left(\mu \mathrm{g} \mathrm{g}^{-1}\right)$ \\
\hline \multirow{14}{*}{2005} & \multirow{14}{*}{ Santos E.C.O. [30] } & \multirow{7}{*}{ São Gabriel da Cachoeira } & \multirow{14}{*}{$\mathrm{AM}$} & 157 & total of population studied & $0 \rightarrow>40$ y.o & G & $0.30-83.11$ & 13.02 \\
\hline & & & & 9 & nd & $0-5$ у.о & C & $1.01-14.40$ & 5.71 \\
\hline & & & & 8 & nd & $6-10$ y.o & C & $2.05-15.00$ & 7.35 \\
\hline & & & & 37 & nd & $11-20$ y.o & $\mathrm{NC}$ & $0.94-22.81$ & 7.346 \\
\hline & & & & 45 & nd & $21-30$ у.о & A & $0.30-59.16$ & 11.67 \\
\hline & & & & 33 & nd & $31-40$ у.о & A & $1.03-60.00$ & 16.56 \\
\hline & & & & 26 & nd & $>40$ y.o & A & $2.41-83.11$ & 22.88 \\
\hline & & \multirow{7}{*}{ Barcelos } & & 242 & total of population studied & $0->40$ у.о & G & $0.07-52.04$ & 9.671 \\
\hline & & & & 17 & nd & $0-5$ у.о & C & $0.83-25.89$ & 7.46 \\
\hline & & & & 25 & nd & $6-10$ у.о & $\mathrm{C}$ & $0.76-27.46$ & 6.85 \\
\hline & & & & 44 & nd & $11-20$ y.o & $\mathrm{NC}$ & $0.07-21.53$ & 7.00 \\
\hline & & & & 62 & nd & $21-30$ у.о & A & $0.25-42.64$ & 9.05 \\
\hline & & & & 27 & nd & $31-40$ y.o & A & $0.23-52.04$ & 12.02 \\
\hline & & & & & & $>40$ y.o & A & $2.60-32.86$ & 12.67 \\
\hline \multirow{4}{*}{2005} & \multirow{4}{*}{ Tavares L.M.B. [31] } & \multirow{4}{*}{$\begin{array}{l}\text { Riverine communities of Bocas de } \\
\text { Conchas, Cuiabá Mirim, Estirão } \\
\text { Cumprido and Porto Brandão located } \\
\text { near of Barão de Melgaço } \\
\text { Barão de Melgaço }\end{array}$} & \multirow{4}{*}{ MT } & & & & & & \\
\hline & & & & 72 & riverine children & $3-7$ y.o & C & $0.58-17.14$ & 5.37 \\
\hline & & & & & & & & & \\
\hline & & & & 114 & urban children & 3-7 y.o & $\mathrm{C}$ & $0.38-7.57$ & 2.08 \\
\hline \multirow{10}{*}{2005} & \multirow{10}{*}{$\begin{array}{l}\text { Klautau-Guimaraes } \\
\text { M.N. [32] }\end{array}$} & \multirow{10}{*}{ Teles Pires } & \multirow{10}{*}{ PA } & 65 & $\begin{array}{l}\text { total of population studied } \\
\text { (Kayabi indigenous) }\end{array}$ & $0 \rightarrow 61$, mean 24.53 y.o & I & nd & 14.75 \\
\hline & & & & 33 & Kayabi indigenous & $0-20$ y.o & I & nd & 17.86 \\
\hline & & & & 25 & Kayabi indigenous & $21-40$ у.о & I & nd & 11.97 \\
\hline & & & & 5 & Kayabi indigenous & $41-60$ y.o & I & nd & 14.35 \\
\hline & & & & 2 & Kayabi indigenous & $>61$ y.o & I & nd & 15.17 \\
\hline & & & & 117 & $\begin{array}{l}\text { total of population studied } \\
\text { (Munduruku indigenous) }\end{array}$ & $0->61$, mean 30.90 y.o & I & nd & nd \\
\hline & & & & 34 & Munduruku indigenous & $0-20$ y.o & I & nd & 4.26 \\
\hline & & & & 56 & Munduruku indigenous & $21-40$ y.o & I & nd & 3.65 \\
\hline & & & & 20 & Munduruku indigenous & $41-60$ y.o & I & nd & 3.75 \\
\hline & & & & 7 & Munduruku indigenous & $>61$ y.o & I & nd & 3.72 \\
\hline \multirow{9}{*}{2006} & \multirow{9}{*}{ Alves M.F.A. [33] } & total (all the riverine populations studied) & \multirow{9}{*}{$\mathrm{AM}$} & 105 & total of adult studied population & $18-50$, mean 32 y.o & A & nd & 35.4 \\
\hline & & Mariuá (Negro River) & & 3 & adults & nd & A & nd & 24.9 \\
\hline & & Marará (Negro River) & & 17 & adults & nd & A & nd & 27.3 \\
\hline & & Piloto (Negro River) & & 25 & adults & nd & A & nd & 33.2 \\
\hline & & Ponta da Terra (Cuiuni River) & & 12 & adults & nd & A & nd & 38.2 \\
\hline & & São Luiz (Negro River) & & 21 & adults & nd & A & nd & 41.4 \\
\hline & & Cumaru (Negro River) & & 16 & adults & nd & A & nd & 43.7 \\
\hline & & Baturité (Negro River) & & 11 & adults & nd & A & nd & 33 \\
\hline & & Manaus & & 105 & total of studied population & $18-50$, mean 28 y.o & A & nd & 1.0 \\
\hline \multirow{11}{*}{2006} & \multirow{11}{*}{ Bastos W.R. [34] } & total (all the populations studied) & \multirow{11}{*}{ RO } & 713 & total of population studied & nd & nd & $5.99-150$ & 15.22 \\
\hline & & Calama & & 34 & nd & nd & nd & $0.50-22.48$ & 9.02 \\
\hline & & Boa Vitoria & & 3 & nd & nd & nd & $10.86-17.05$ & 13.82 \\
\hline & & Cujubim & & 12 & nd & nd & nd & $1.55-14.67$ & 6.30 \\
\hline & & Firmesa & & 4 & nd & nd & nd & $9.40-14.80$ & 11.21 \\
\hline & & Itacoa & & 6 & nd & nd & nd & $5.28-16.00$ & 11.97 \\
\hline & & Nazaré & & 64 & nd & nd & nd & $0.63-22.60$ & 10.65 \\
\hline & & Papagaios & & 13 & nd & nd & nd & $4.76-27.22$ & 13.72 \\
\hline & & Santa Rosa & & 19 & nd & nd & nd & $7.68-20.78$ & 13.99 \\
\hline & & São Carlos & & 15 & nd & nd & nd & $1.84-22.83$ & 9.51 \\
\hline & & Terra Caida & & 7 & nd & nd & nd & $5.01-14.61$ & 9.61 \\
\hline
\end{tabular}


Table 2. Cont.

\begin{tabular}{|c|c|c|c|c|c|c|c|c|c|}
\hline Year & Author & Locality & State & $n$ & $\begin{array}{l}\text { Type of Population According to } \\
\text { the Author }\end{array}$ & Population Specificity & $\begin{array}{l}\text { Type of the Population According } \\
\text { to the Present Study }\end{array}$ & Range $\mathrm{Hg}\left(\mu \mathrm{g} \mathrm{g}^{-1}\right)$ & Mean $\mathrm{Hg}\left(\mu \mathrm{g} \mathrm{g}^{-1}\right)$ \\
\hline \multirow{35}{*}{2006} & \multirow{35}{*}{ Bastos W.R. [34] } & Sto Antônio do Pau Queimado & RO & 14 & nd & nd & nd & $5.87-26.86$ & 14.69 \\
\hline & & Puruzinho & \multirow{34}{*}{ AM } & 28 & nd & nd & nd & $4.57-28.27$ & 14.83 \\
\hline & & Livramento & & 15 & nd & nd & nd & $18.96-63.54$ & 36.89 \\
\hline & & Valparaiso & & 21 & nd & nd & nd & $2.98-82.38$ & 18.93 \\
\hline & & Auxiliadora & & 34 & nd & nd & nd & $1.12-22.78$ & 9.34 \\
\hline & & Curralinho & & 5 & nd & nd & nd & $10.70-34.49$ & 19.69 \\
\hline & & Nazaré do Retiro & & 15 & nd & nd & nd & $9.69-24.77$ & 17.90 \\
\hline & & Novos Prazeres & & 20 & nd & nd & nd & $2.77-24.28$ & 11.90 \\
\hline & & São Pedro & & 14 & nd & nd & nd & $6.61-28.00$ & 15.77 \\
\hline & & Barreiras do Manicoré & & 9 & nd & nd & nd & $1.45-23.04$ & 10.82 \\
\hline & & Cachoeirinha & & 14 & nd & nd & nd & $1.54-37.22$ & 14.74 \\
\hline & & São Lazaro & & 6 & nd & nd & nd & $2.50-23.37$ & 9.48 \\
\hline & & Maraca II & & 6 & nd & nd & nd & $8.57-15.69$ & 11.37 \\
\hline & & Vista Nova & & 4 & nd & nd & nd & $21.40-28.54$ & 25.69 \\
\hline & & Vista Alegre & & 17 & nd & nd & nd & $7.28-26.28$ & 16.02 \\
\hline & & Bom Suspiro & & 12 & nd & nd & nd & $6.43-30.06$ & 16.29 \\
\hline & & Carara & & 39 & nd & nd & nd & $4.18-34.71$ & 18.13 \\
\hline & & Miriti & & 16 & nd & nd & nd & $6.70-50.37$ & 22.34 \\
\hline & & São Sebastiao (Lago Lucio) & & 17 & nd & nd & nd & $6.61-18.52$ & 12.84 \\
\hline & & Boca do Carapanatuba & & 18 & nd & nd & nd & $3.43-19.22$ & 10.45 \\
\hline & & São Sebastiao do Tapuru & & 18 & nd & nd & nd & $20.43-150.00$ & 62.76 \\
\hline & & Moanenses & & 13 & nd & nd & nd & $3.26-20.49$ & 12.73 \\
\hline & & Três Casas & & 9 & nd & nd & nd & $5.62-70.70$ & 33.07 \\
\hline & & Boa Ventura & & 7 & nd & nd & nd & $4.73-35.79$ & 16.55 \\
\hline & & Auara Grande & & 19 & nd & nd & nd & $6.21-24.98$ & 15.97 \\
\hline & & Fazenda Tabocal & & 2 & nd & nd & nd & $0.50-1.50$ & 1.00 \\
\hline & & Remanso & & 12 & nd & nd & nd & $8.36-29.02$ & 18.16 \\
\hline & & Arapapa & & 7 & nd & nd & nd & 10.43-21.33 & 16.56 \\
\hline & & Axinim & & 13 & nd & nd & nd & $3.27-23.02$ & 8.65 \\
\hline & & Espirito Santo & & 18 & nd & nd & nd & $3.51-21.28$ & 12.47 \\
\hline & & Santa Maria & & 7 & nd & nd & nd & $6.70-16.84$ & 9.28 \\
\hline & & Caicara & & 23 & nd & nd & nd & $1.94-17.98$ & 10.04 \\
\hline & & Paquique & & 6 & nd & nd & nd & $7.49-11.57$ & 9.23 \\
\hline & & Uricurituba & & 46 & nd & nd & nd & $0.36-19.12$ & 9.09 \\
\hline & & Santa Rosa II & & 12 & nd & nd & nd & $5.81-16.89$ & 11.65 \\
\hline 2006 & Fillion M. [35] & $\begin{array}{l}\text { Tapajóśs (São Luiz do Tapajós, } \\
\text { Nova Canaã, Santo Antônio, } \\
\text { Mussum, Vista Alegre, } \\
\text { Açaituba) }\end{array}$ & PA & 251 & adults & $15-89$, mean 35.2 y.o & A & $0.21-77.2$ & 17.8 \\
\hline \multirow[t]{3}{*}{2007} & Passos C.J. [36] & $\begin{array}{l}\text { Tapajós (São Luiz do Tapajós, } \\
\text { Nova Canaã, Santo Antônio, } \\
\text { Ipaupixuna, Novo Paraisoo, } \\
\text { Teca, Timbó, Açaituba, } \\
\text { Campo Alegre, Sumauma, } \\
\text { Vista Alegre, Mussum, } \\
\text { Santa Cruz) } \\
\end{array}$ & PA & 449 & adults & $15-89$, mean 38.6 y.o & A & $0.2-58.3$ & 16.8 \\
\hline & \multirow{5}{*}{ Pinheiro M.C.N. [37] } & & \multirow{5}{*}{ PA } & 8 & children & $0-1$ у.о & C & $0.39-4.66$ & 1.11 \\
\hline & & Panacauera & & $\begin{array}{l}13 \\
15\end{array}$ & $\begin{array}{l}\text { children } \\
\text { children }\end{array}$ & $\begin{array}{l}2-6 \text { y.o } \\
7-12 \text { y.o }\end{array}$ & $\begin{array}{l}\mathrm{C} \\
\mathrm{C}\end{array}$ & $\begin{array}{l}0.65-5.16 \\
0.86-9.46\end{array}$ & $\begin{array}{l}2.27 \\
2.99\end{array}$ \\
\hline \multirow{3}{*}{2007} & & & & 17 & children & $0-1$ y.o & C & $1.80-15.70$ & 5.35 \\
\hline & & Barreiras (Tapajós Basin) & & 45 & children & $2-6$ y.o & C & $1.43-23.60$ & 6.21 \\
\hline & & & & 22 & children & $7-12$ у.о & C & $1.63-14.50$ & 6.72 \\
\hline
\end{tabular}


Table 2. Cont

\begin{tabular}{|c|c|c|c|c|c|c|c|c|c|}
\hline Year & Author & Locality & State & $n$ & $\begin{array}{l}\text { Type of Population According to } \\
\text { the Author }\end{array}$ & Population Specificity & $\begin{array}{l}\text { Type of the Population According } \\
\text { to the Present Study }\end{array}$ & Range $\mathrm{Hg}\left(\mu \mathrm{g} \mathrm{g}^{-1}\right)$ & Mean $\mathrm{Hg}\left(\mu \mathrm{g} \mathrm{g}^{-1}\right)$ \\
\hline & & São Luiz do Tapajós & & $\begin{array}{l}11 \\
23 \\
14\end{array}$ & $\begin{array}{l}\text { children } \\
\text { children } \\
\text { children }\end{array}$ & $\begin{array}{l}0-1 \text { y.o } \\
2-6 \text { y.o } \\
7-12 \text { y.o }\end{array}$ & $\begin{array}{l}\mathrm{C} \\
\mathrm{C} \\
\mathrm{C}\end{array}$ & $\begin{array}{l}1.99-30.30 \\
2.76-53.80 \\
1.34-38.80\end{array}$ & $\begin{array}{l}5.97 \\
13.22 \\
10.83\end{array}$ \\
\hline 2008 & Passos C.J.S. [38] & $\begin{array}{l}\text { Tapajós (São Luiz do Tapajós, } \\
\text { Nova Canaã, Santo Antônio, } \\
\text { Vista Alegre, Mussum, } \\
\text { Açaituba) }\end{array}$ & PA & 256 & adults & $15-89$, mean 35.3 у.о & A & $0.2-58.3$ & 17.9 \\
\hline 2009 & Fillion M. [39] & $\begin{array}{l}\text { Tapajós (São Luiz do Tapajós, } \\
\text { Nova Canaã, Santo Antônio, } \\
\text { Mussum, Vista Alegre, } \\
\text { Açaituba, Santa Cruz, } \\
\text { Sumauma, Campo Alegre, } \\
\text { Ipaupixuna, Novo Paraiso, } \\
\text { Curi-Teca, Curi-Timbó) }\end{array}$ & PA & 456 & adults & $15->65$ y.o & A & $0.2-77.7$ & 17.8 \\
\hline 2010 & Grotto D. [40] & Tapajós & PA & $\begin{array}{l}108 \\
54 \\
54\end{array}$ & $\begin{array}{l}\text { total of population studied } \\
\text { men } \\
\text { women }\end{array}$ & $\begin{array}{l}\text { mean } 41.1 \text { y.o } \\
\text { nd } \\
\text { nd }\end{array}$ & $\begin{array}{l}\text { nd } \\
\mathrm{M} \\
\mathrm{W}\end{array}$ & $\begin{array}{l}1-57.8 \\
\text { nd } \\
\text { nd }\end{array}$ & $\begin{array}{l}13.7 \\
11.5 \\
8.8\end{array}$ \\
\hline 2010 & Bortoli M.C. [41] & Novo Airão & $\mathrm{AM}$ & 55 & women & mean 32.3 y.o & $\mathrm{W}$ & $0.04-18.67$ & 5.67 \\
\hline 2012 & Barcelos G.R.M. [42] & Tapajós & PA & 144 & adults & $15-83$, mean 43 y.o & A & $1-43.3$ & 10.4 \\
\hline 2012 & Dutra M.D.S. [43] & Itaituba & PA & 90 & children & $\begin{array}{l}\text { population from urban } \\
\text { area, samples collected } \\
\text { in } 2004\end{array}$ & C & nd & 1.01 \\
\hline & & & & 47 & children & $\begin{array}{l}\text { population rrom urban } \\
\text { area, samples collected } \\
\text { in } 2006\end{array}$ & c & nd & 1.18 \\
\hline & & & & 90 & children & $\begin{array}{l}\text { population from urban } \\
\text { area, samples collected } \\
\text { in } 2010\end{array}$ & C & nd & 1.18 \\
\hline 2012 & Farias L.A. [44] & Manaus & AM & 201 & children & $2-7$ y.o & C & $0.02-34.4$ & 1.93 \\
\hline 2013 & Khoury E.D.T. [45] & $\begin{array}{c}\text { Barreiras } \\
\text { São Luiz do Tapajós } \\
\text { Furo do Maracujá }\end{array}$ & PA & $\begin{array}{l}78 \\
30 \\
49\end{array}$ & $\begin{array}{l}\text { general population } \\
\text { general population } \\
\text { general population }\end{array}$ & $\begin{array}{l}13-53 \text { y.o } \\
13-53 \text { y.o } \\
13-53 \text { y.o }\end{array}$ & $\begin{array}{l}G \\
G \\
G\end{array}$ & $\begin{array}{l}\text { nd } \\
\text { nd } \\
\text { nd }\end{array}$ & $\begin{array}{l}8.66 \\
9.19 \\
0.73\end{array}$ \\
\hline 2014 & Rocha A.V. [46] & $\begin{array}{l}\text { Demarcação-Machado River } \\
\text { Gleba do Rio Preto }\end{array}$ & RO & $\begin{array}{l}10 \\
10\end{array}$ & $\begin{array}{l}\text { children } \\
\text { children }\end{array}$ & $\begin{array}{l}3-9 \text { y.o } \\
3-9 \text { y.o }\end{array}$ & $\begin{array}{l}\mathrm{C} \\
\mathrm{C}\end{array}$ & $\begin{array}{l}\text { nd } \\
\text { nd }\end{array}$ & $\begin{array}{l}3.57 \\
6.24\end{array}$ \\
\hline 2015 & Faial K. [47] & Itaituba & PA & $\begin{array}{c}6 \\
6 \\
10 \\
6 \\
7 \\
4 \\
\text { nd } \\
3 \\
5 \\
1 \\
1 \\
1 \\
3\end{array}$ & $\begin{array}{l}\text { male } \\
\text { male } \\
\text { male } \\
\text { male } \\
\text { male } \\
\text { male } \\
\text { male } \\
\text { male } \\
\text { male } \\
\text { male } \\
\text { male } \\
\text { male } \\
\text { male }\end{array}$ & $\begin{array}{l}0-2 \text { y.o } \\
3-5 \text { y.o } \\
6-10 \text { y.o } \\
11-15 \text { y.o } \\
16-20 \text { y.o } \\
21-25 \text { y.o } \\
26-30 \text { y.o } \\
31-35 \text { y.o } \\
36-40 \text { y.o } \\
41-45 \text { y.o } \\
46-50 \text { y.o } \\
51-55 \text { y.o } \\
56-60 \text { yo }\end{array}$ & $\begin{array}{l}\text { C } \\
\text { C } \\
\text { C } \\
\text { C } \\
\text { A } \\
\text { A } \\
\text { A } \\
\text { A } \\
\text { A } \\
\text { A } \\
\text { A } \\
\text { A }\end{array}$ & $\begin{array}{c}4.14-9.79 \\
16.01-23.80 \\
12.59-24.93 \\
5.83-15.57 \\
17.82-24.16 \\
9.42-20.09 \\
\text { nd } \\
8.55-10.83 \\
10.92-20.03 \\
14.81-14.81 \\
2.07-2.07 \\
13.89-13.89 \\
14.00-14.16\end{array}$ & $\begin{array}{c}6.85 \\
19.57 \\
18.58 \\
13.08 \\
20.87 \\
15.52 \\
\text { nd } \\
9.69 \\
15.29 \\
14.81 \\
2.07 \\
13.89 \\
14.08\end{array}$ \\
\hline
\end{tabular}


Table 2. Cont.

\begin{tabular}{|c|c|c|c|c|c|c|c|c|c|}
\hline Year & Author & Locality & State & $n$ & $\begin{array}{l}\text { Type of Population According to } \\
\text { the Author }\end{array}$ & Population Specificity & $\begin{array}{l}\text { Type of the Population According } \\
\text { to the Present Study }\end{array}$ & Range $\mathrm{Hg}\left(\mu \mathrm{g} \mathrm{g}^{-1}\right)$ & Mean $\mathrm{Hg}\left(\mu \mathrm{g} \mathrm{g}^{-1}\right)$ \\
\hline 2015 & Faial K. [47] & Itaituba & PA & $\begin{array}{l}5 \\
4 \\
7 \\
7 \\
8 \\
5 \\
14 \\
7 \\
8 \\
3 \\
3 \\
3 \\
4 \\
1 \\
9\end{array}$ & $\begin{array}{c}\text { male } \\
\text { female } \\
\text { female } \\
\text { female } \\
\text { female } \\
\text { female } \\
\text { female } \\
\text { female } \\
\text { female } \\
\text { female } \\
\text { female } \\
\text { female } \\
\text { female } \\
\text { female } \\
\text { female }\end{array}$ & $\begin{array}{l}>60 \text { y.o } \\
0-2 \text { y.o } \\
3-5 \text { y.o } \\
6-10 \text { y.o } \\
11-15 \text { y.o } \\
16-20 \text { y.o } \\
21-25 \text { y.o } \\
26-30 \text { y.o } \\
31-35 \text { y.o } \\
36-40 \text { y.o } \\
41-45 \text { y.o } \\
46-50 \text { y.o } \\
51-55 \text { y.o } \\
56-60 \text { y.o } \\
>60 \text { y.o }\end{array}$ & $\begin{array}{l}\mathrm{A} \\
\mathrm{C} \\
\mathrm{C} \\
\mathrm{C} \\
\mathrm{C} \\
\mathrm{A} \\
\mathrm{A} \\
\mathrm{A} \\
\mathrm{A} \\
\mathrm{A} \\
\mathrm{A} \\
\mathrm{A} \\
\mathrm{A} \\
\mathrm{A} \\
\mathrm{A}\end{array}$ & $\begin{array}{c}12.04-21.47 \\
8.25-12.89 \\
12.20-19.29 \\
11.46-23.21 \\
8.41-21.71 \\
13.52-22.01 \\
6.33-17.66 \\
4.84-10.6 \\
4.95-20.78 \\
7.30-15.73 \\
14.69-23.02 \\
7.31-14.98 \\
15.52-18.52 \\
14.29-14.29 \\
14.60-27.02\end{array}$ & $\begin{array}{l}14.57 \\
10.38 \\
15.83 \\
15.84 \\
13.12 \\
16.74 \\
11.22 \\
7.16 \\
13.52 \\
11.09 \\
18.62 \\
10.27 \\
17.02 \\
14.29 \\
20.39\end{array}$ \\
\hline 2015 & Castilhos Z. [48] & $\begin{array}{l}\text { São Chico } \\
\text { Creporizinho }\end{array}$ & $\mathrm{PA}$ & $\begin{array}{l}172 \\
146 \\
\end{array}$ & $\begin{array}{l}\text { nd } \\
\text { nd }\end{array}$ & $\begin{array}{l}\text { nd } \\
\text { nd }\end{array}$ & $\begin{array}{l}\text { nd } \\
\text { nd }\end{array}$ & $\begin{array}{l}0.14-35.90 \\
0.23-10.49\end{array}$ & $\begin{array}{l}3.44 \\
2.25\end{array}$ \\
\hline 2015 & Hoshino A. [49] & Lago do Puruzinho & $\mathrm{AM}$ & 58 & general population & $1-47$, mean 17.3 y.o & G & nd & 12.78 \\
\hline 2016 & Rocha A.V. [50] & Porto Velho & RO & 200 & women & $18-48$, mean 26.60 y.o & $\mathrm{W}$ & nd & 0.60 \\
\hline 2016 & Carvalho L.V.B. [51] & $\begin{array}{l}\text { Belmont } \\
\text { Cunia }\end{array}$ & RO & $\begin{array}{l}42 \\
52\end{array}$ & $\begin{array}{l}\text { nd } \\
\text { nd }\end{array}$ & $\begin{array}{l}\text { mean } 11.3 \text { y.o } \\
\text { mean } 11.3 \text { y.o }\end{array}$ & $\begin{array}{l}\text { nd } \\
\text { nd }\end{array}$ & $\begin{array}{l}\text { nd } \\
\text { nd }\end{array}$ & $\begin{array}{l}2.71 \\
7.18\end{array}$ \\
\hline & & & & 11.827 & & & & & \\
\hline
\end{tabular}


There was a predominance of studies that were performed in the State of Para (47\%) $[22,23,25-27,29,35-40,42,43,45,47,48]$. Some studies had been performed in the State of Amazonas (22\%) [17,19,24,30,33,41,44,49], Rondônia (14\%) [18,28,46,50,51], Mato Grosso (8\%) [21,31,32] and Amapá (3\%) [20]. Two studies were performed using samples from populations from two different States: Pará and Rondônia (3\%) [16] and Rondônia and Amazonas (3\%) [34].

The results shows that general population presented the highest mean level of $\mathrm{Hg}$ exposure (29.59 $\mathrm{\mu g} \mathrm{g}^{-1}$, ranging from 0.73 to $97.44 \mu \mathrm{g} \mathrm{g}^{-1}$ ), followed by adult population (21.08 $\mu \mathrm{g} \mathrm{g}^{-1}$, ranging from 1.00 to $\left.43.70 \mu \mathrm{g} \mathrm{g}^{-1}\right)$, not determined $\left(14.60 \mu \mathrm{g} \mathrm{g}{ }^{-1}\right.$, ranging from 1.00 to $\left.62.76 \mu \mathrm{g} \mathrm{g}^{-1}\right)$, men population $\left(11.25 \mu \mathrm{g} \mathrm{g}^{-1}\right.$, ranging from 11.00 to $\left.11.50 \mu \mathrm{g} \mathrm{g}^{-1}\right)$, children population $\left(7.95 \mu \mathrm{g} \mathrm{g}^{-1}\right.$, ranging from 1.11 to $\left.22.00 \mu \mathrm{g} \mathrm{g}^{-1}\right)$, women population $\left(7.66 \mu \mathrm{g} \mathrm{g}^{-1}\right.$, ranging from 0.60 to $14.30 \mu \mathrm{g} \mathrm{g}^{-1}$ ) and Indigenous population (6.95 $\mathrm{gg} \mathrm{g}^{-1}$, ranging from 4.90 to $\left.8.37 \mu \mathrm{g} \mathrm{g}^{-1}\right)$ (Figure 2).

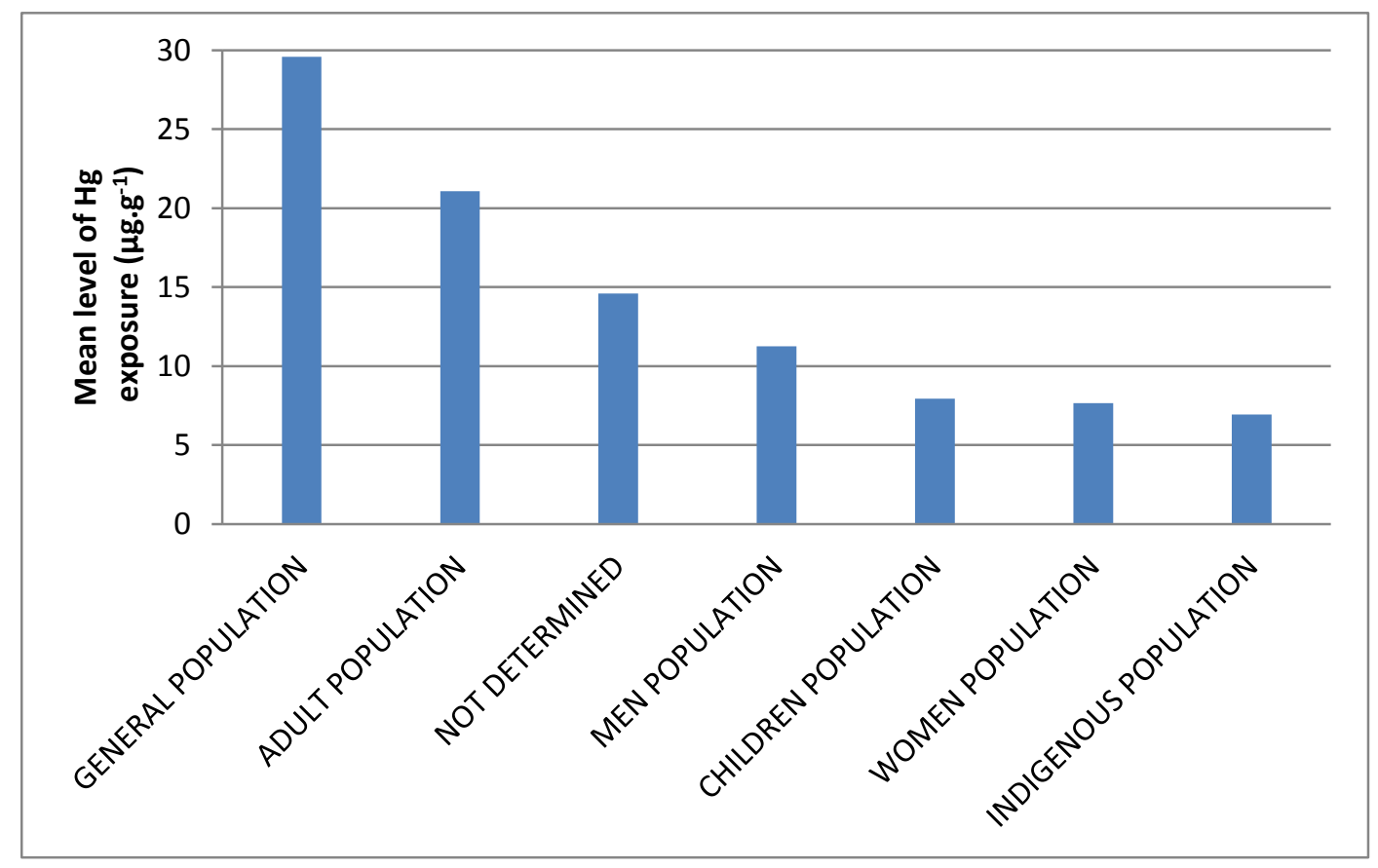

Figure 2. Mean level $\left(\mu \mathrm{g} \mathrm{g}^{-1}\right)$ of $\mathrm{Hg}$ exposure in populations of Brazilian Amazon according to the present study.

The analysis of the georeferencing data is presented as a map which shows a geospatial distribution of the populations and their respective degree of $\mathrm{Hg}$ exposure. The results show that most of the studies were performed along the margins of the Amazonian rivers and that most of these populations are highly exposed (Figure 3). 


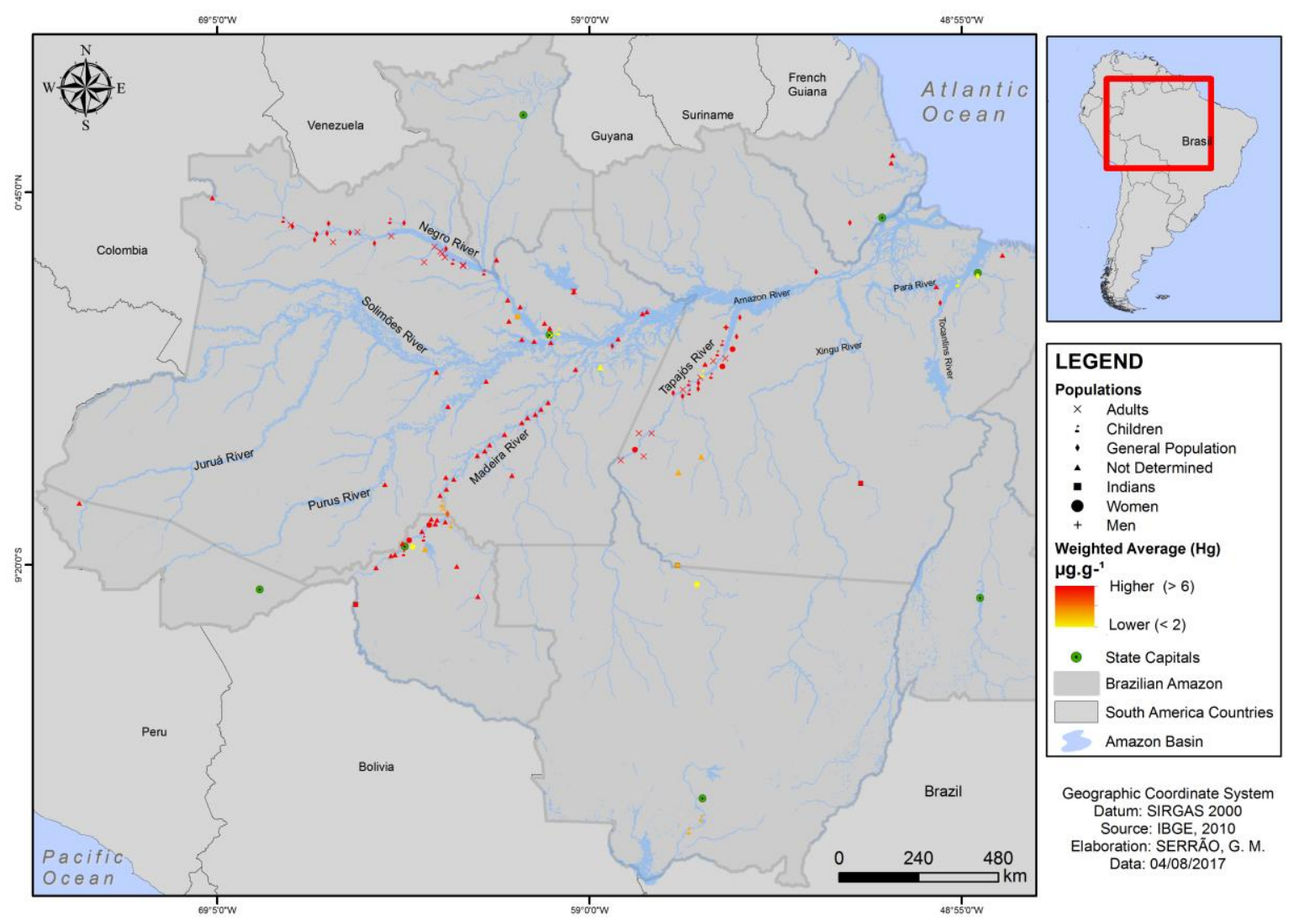

Figure 3. The georeferencing results showing a geospatial distribution of the Brazilian Amazonian populations and their respective degree of $\mathrm{Hg}$ exposure on hair.

\section{Discussion}

The theme of $\mathrm{Hg}$ exposure in Amazonian population is an intriguing form of environmental contamination for some reasons: (1) the mean level of $\mathrm{Hg}$ exposure in Amazonian population usually exceed the normal limit preconized by WHO [24,30,33,52-54]; (2) there is a dichotomy in the clinical findings in different Amazonian populations, where some studies associate $\mathrm{Hg}$ exposure with the development of clinical symptoms [35,55-57] while others do not [45,58-61] and (3) even "non-exposed" populations are at risk of $\mathrm{Hg}$ effects [58].

The present study performed a systematic review of $\mathrm{Hg}$ exposure in Brazilian Amazon population. The results show that the majority of the studies were performed by Brazilian Institutions and researchers, reflecting the low international insertion despite that $\mathrm{Hg}$ is a global problem [62]. The high number of studies that were performed in the State of Pará is in agreement with the high prevalence of gold miners in the region (legal and illegal miners), especially in the Tapajós Basin [26,63].

The analysis shows that most of the studies were performed along the margins of important rivers, such as Negro, Tapajós and Madeira Rivers. The results show that all the populations presented mean levels above $6 \mu \mathrm{g} \mathrm{g}^{-1}$ of $\mathrm{Hg}$ on hair and that general population, adults, not determined and men presented mean levels above $10 \mu \mathrm{g} \mathrm{g}^{-1}$ of $\mathrm{Hg}$ on hair. Thus, the findings support the idea that Amazonian population present (along the period of time covered by this study) mean levels of $\mathrm{Hg}$ above the normal limit preconized by WHO (1-2 $\mu \mathrm{g} \mathrm{g}^{-1}$ and levels above $10 \mu \mathrm{g} \mathrm{g}^{-1}$ for daily fish consumers) [64]. Although, affirm that this population is under risk of $\mathrm{Hg}$ effect is premature. Discussions about this issue should be evaluated based on other studies.

As future perspectives, the present study suggests an implementation of a strategic plan for the region in order to promote health and benefit the population. As strategies, we propose:

(1) Increase the technical capacity for $\mathrm{Hg}$ determination in the region; 
(2) Implement an environmental health surveillance program that considerers the Amazonian life style, behavior and ecosystem dynamics;

(3) A follow up program to monitor the $\mathrm{Hg}$ content and health of individuals that presents high levels of $\mathrm{Hg}$ on biological matrices.

\section{Conclusions}

The $\mathrm{Hg}$ exposure in the Amazonian population is a fact. The high level of $\mathrm{Hg}$ on hair revealed by this study shows that this population has been long-term exposed to this metal.

The data reveals that the studies focused on population that lives along the margins of the rivers and, of utmost importance, populations under risk of $\mathrm{Hg}$ exposure from gold mining activities, especially from Tapajós Basin located in the State of Pará.

Author Contributions: Nathália Santos Serrão de Castro and Marcelo de Oliveira Lima were responsible for: (1) Design, analysis and interpretation of data; (2) Article writing and critical review of the intellectual content; (3) Final approval of the version to be published; (4) All aspects of the work, ensuring the accuracy and integrity of any part of the work.

Conflicts of Interest: The authors declare no conflict of interest.

Disclaimer: The opinions or assertions contained herein are the private views of the author(s) and are not to be construed as official or to reflect the views of the Evandro Chagas Institute or the Metropolitan College of Amazon.

\section{References}

1. Ekino, S.; Susa, M.; Ninomiya, T.; Imamura, K.; Kitamura, T. Minamata disease revisited: An update on the acute and chronic manifestations of methyl mercury poisoning. J. Neurol. Sci. 2007, 262, 131-144. [CrossRef] [PubMed]

2. Ninomiya, T.; Ohmori, H.; Hashimoto, K.; Tsuruta, K.; Ekino, S. Expansion of methylmercury poisoning outside of Minamata: An epidemiological study on chronic methylmercury poisoning outside of Minamata. Environ. Res. 1995, 70, 47-50. [CrossRef] [PubMed]

3. Ninomiya, T.; Imamuraa, K.; Kuwahatab, M.; Kindaichid, M.; Susac, M.; Ekino, S. Reappraisal of somatosensory disorders in methylmercury poisoning. Neurotoxicol. Teratol. 2005, 27, 643-653. [CrossRef] [PubMed]

4. Yorifuji, T.; Tsuda, T.; Takao, S.; Harada, M. Long-term exposure to methylmercury and neurologic signs in Minamata and neighboring communities. Epidemiology 2008, 19, 3-9. [CrossRef] [PubMed]

5. Maruyama, K.; Yorifuji, T.; Tsuda, T.; Sekikawa, T.; Nakadaira, H.; Saito, H. Methyl mercury exposure at Niigata, Japan: Results of neurological examinations of 103 adults. J. Biomed. Biotechnol. 2012, 2012, 635075. [CrossRef] [PubMed]

6. $\quad$ Karagas, M.R.; Choi, A.L.; Oken, E.; Horvat, M.; Schoeny, R.; Kamai, E.; Cowell, W.; Grandjean, P.; Korrick, S. Evidence on the human health effects of low-level methylmercury exposure. Environ. Health Perspect. 2012, 120, 799-807. [CrossRef] [PubMed]

7. Holmes, P.; James, K.A.F.; Levy, L.S. Is low-level environmental mercury exposure of concern to human health? Sci. Total Environ. 2009, 408, 171-182. [CrossRef] [PubMed]

8. Grandjean, P.; White, R.F.; Weihe, P.; Jørgensen, P.J. Neurotoxic risk caused by stable and variable exposure to methylmercury from seafood. Ambul. Pediatr. 2003, 3, 18-23. [CrossRef]

9. Grandjean, P.; Weihe, P.; White, R.F.; Debes, F. Cognitive performance of children prenatally exposed to 'safe' levels of methylmercury. Environ. Res. 1998, 77, 165-172. [CrossRef] [PubMed]

10. Amin-Zaki, L.; Elhassani, S.; Majeed, M.A.; Clarkson, T.W.; Doherty, R.A.; Greenwood, M. Intra-uterine Methylmercury Poisoning in Iraq. Pediatrics 1974, 54, 587-595. [PubMed]

11. Yorifuji, T.; Kashima, S.; Tsuda, T.; Harada, M. What has methylmercury in umbilical cords told us?-Minamata disease. Sci. Total Environ. 2009, 408, 272-276. [CrossRef] [PubMed]

12. Airey, D. Mercury in human hair due to environment and diet: A review. Environ. Health Perspect. 1983, 52, 303-316. [CrossRef] [PubMed]

13. Gosselin, N.H.; Brunet, R.C.; Carrier, G.; Bouchard, M.; Feeley, M. Reconstruction of methylmercury intakes in indigenous populations from biomarker data. J. Expo. Sci. Environ. Epidemiol. 2006, 16, 19-29. [CrossRef] [PubMed] 
14. Kempson, I.M.; Lombi, E. Hair analysis as a biomonitor for toxicology, disease and health status. Chem. Soc. Rev. 2011, 40, 3915-3940. [CrossRef] [PubMed]

15. Grandjean, P.; Jørgensen, P.J.; Weihe, P. Validity of mercury exposure biomarkers. In Biomarkers of Environmentally Associated Disease; Samuel, H., Wilson, W.A.S., Eds.; CRC Press: Boca Raton, FL, USA, 2002; pp. 235-247.

16. Barbosa, A.C.; Silva, S.R.L.; Dórea, J.G. Concentration of mercury in hair of indigenous mothers and infants from the Amazon Basin. Arch. Environ. Contam. Toxicol. 1998, 34, 100-105. [CrossRef] [PubMed]

17. Kehrig, H.A.; Malm, O.; Akagi, H.; Guimarães, J.R.; Torres, J.P. Methylmercury in fish and hair samples from the Balbina Feservoir, Brazilian Amazon. Environ. Res. 1998, 77, 84-90. [CrossRef] [PubMed]

18. Barbosa, A.C.; Dórea, J.G. Indices of mercury contamination during breast feeding in the Amazon Basin. Environ. Toxicol. Pharmacol. 1998, 6, 71-79. [CrossRef]

19. Silva-Forsberg, M.C.; Forsberg, B.R.; Zeldemann, V.K. Mercury contamination in humans linked to river chemistry in the Amazon Basin. Ambio 1999, 28, 519-521.

20. Guimaraes, J.R.D.; Forti, M.C. Mercury in human and environmental samples from two lakes in Amapa, Brazilian Amazon. Ambio 1999, 28, 296-301.

21. Hacon, S.; Yokoo, E.; Valente, J.; Campos, R.C.; Da Silva, V.A.; de Menezes, A.C.C.; De Moraes, L.P.; Ignotti, E. Exposure to mercury in pregnant women from Alta Floresta-Amazon Basin, Brazil. Environ. Res. 2000, 84, 204-210. [CrossRef] [PubMed]

22. De Oliveira Santos, E.C.; de Jesus, I.M.; da Silva Brabo, E.; Loureiro, E.C.B.; da Silva Mascarenhas, A.F.; Weirich, J.; Câmara, V.D.M.; Cleary, D. Mercury exposures in riverside Amazon communities in Pará, Brazil. Environ. Res. 2000, 84, 100-107. [CrossRef]

23. Dolbec, J.; Mergler, D.; Sousa Passos, C.J.; Sousa de Morais, S.; Lebel, J. Methylmercury exposure affects motor performance of a riverine population of the Tapajós river, Brazilian Amazon. Int. Arch. Occup. Environ. Health 2000, 73, 195-203. [CrossRef] [PubMed]

24. Barbosa, A.C.; Jardim, W.; Dórea, J.G.; Fosberg, B.; Souza, J. Hair mercury speciation as a function of gender, age, and body mass index in inhabitants of the Negro River Basin, Amazon, Brazil. Arch. Environ. Contam. Toxicol. 2001, 40, 439-444. [PubMed]

25. Harada, M.; Nakanishi, J.; Yasoda, E.; Maria da Conceicâo, N.P.; Oikawa, T.; de Assis Guimarâes, G.; da silva Cardoso, B.; Kizaki, T.; Ohno, H. Mercury pollution in the Tapajos River Basin, Amazon mercury level of head hair and health effects. Environ. Int. 2001, 27, 285-290. [CrossRef]

26. Crompton, P.; Ventura, A.M.; de Souza, J.M.; Santos, E.; Strickland, G.T.; Silbergeld, E. Assessment of mercury exposure and malaria in a Brazilian Amazon riverine community. Environ. Res. 2002, 90, 69-75. [CrossRef] [PubMed]

27. Passos, C.J.; Mergler, D.; Gaspar, E.; Morais, S.; Lucotte, M.; Larribe, F.; Davidson, R.; de Grosbois, S. Eating tropical fruit reduces mercury exposure from fish consumption in the Brazilian Amazon. Environ. Res. 2003, 93, 123-130. [CrossRef]

28. Santos, E.C.O.; Câmara, V.M.; Brabo, E.S.; Loureiro, E.C.B.; Jesus, I.M.; Fayal, K.; Sagica, F. Mercury exposure evaluation among Pakaanóva Indians, Amazon Region, Brazil. Cad. Saude Publica 2003, 19, 199-206. [CrossRef] [PubMed]

29. Dórea, J.G.; de Souza, J.R.; Rodrigues, P.; Ferrari, I.; Barbosa, A.C. Hair mercury (signature of fish consumption) and cardiovascular risk in Munduruku and Kayabi Indians of Amazonia. Environ. Res. 2005, 97, 209-219. [CrossRef] [PubMed]

30. Santos, E.C.O.; Sá, G.C.; Jesus, I.M.; Brabo, E.S.; Câmara, V.M.; Lima, M.O.; Faial, K.F.; Mendes, R.A.; Mascarenhas, A.F.S. Mercury in the Negro river, Brazilian Amazon-Preliminary study of exposure indicators in fish and human populations. Cad. Saúde Coletiva 2005, 13, 225-235.

31. Tavares, L.M.B.; Câmara, V.M.; Malm, O.; Santos, E.C.O. Performance on neurological development tests by riverine children with moderate mercury exposure in Amazonia, Brazil. Cad. Saude Publica 2005, 21, 1160-1167. [CrossRef] [PubMed]

32. Klautau-Guimarães, M.N.; D’Ascenção, R.; Caldart, F.A.; Grisolia, C.K.; Souza, J.R.; Barbosa, A.C.; Cordeiro, C.M.T.; Ferrari, I. Analysis of genetic susceptibility to mercury contamination evaluated through molecular biomarkers in at-risk Amazon Amerindian populations. Genet. Mol. Biol. 2005, 832, 827-832. [CrossRef] 
33. Alves, M.F.A.; Fraijo, N.A.; Barbosa, A.C.; De Lima, D.S.; Souza, J.R.; Dórea, J.G.; Cordeiro, G.W.O. Fish consumption, mercury exposure and serum antinuclear antibody in Amazonians. Int. J. Environ. Health Res. 2006, 16, 255-262. [CrossRef] [PubMed]

34. Bastos, W.R.; Gomes, J.P.O.; Oliveira, R.C.; Almeida, R.; Nascimento, E.L.; Bernadi, J.V.E.; Lacerda, L.D.; Silveira, E.G.; Pfeiffer, W.C. Mercury in the environment and riverside population in the Madeira River Basin, Amazon, Brazil. Sci. Total Environ. 2006, 368, 344-351. [CrossRef] [PubMed]

35. Fillion, M.; Mergler, D.; Passos, C.J.S.; Larribe, F.; Lemire, M.; Guimarães, J.R.D. A preliminary study of mercury exposure and blood pressure in the Brazilian Amazon. Environ. Health 2006, 5, 29. [CrossRef] [PubMed]

36. Passos, C.J.S.; Mergler, D.; Fillion, M.; Lemire, M.; Mertens, F.; Guimarães, J.R.D.; Philibert, A. Epidemiologic confirmation that fruit consumption influences mercury exposure in riparian communities in the Brazilian Amazon. Environ. Res. 2007, 105, 183-193. [CrossRef] [PubMed]

37. Pinheiro, M.C.N.; Cresco-López, M.E.; Vieira, J.L.F.; Oikawa, T.; Guimarães, G.A.; Araújo, C.C.; Amoras, W.W.; Ribeiro, D.R.; Herculano, A.M.; do Nascimento, J.L.M.; et al. Mercury pollution and childhood in Amazon riverside villages. Environ. Int. 2007, 33, 56-61. [CrossRef] [PubMed]

38. Passos, C.J.S.; Silva, D.S.; Lemire, M.; Fillion, M.; Guimarães, J.R.D.; Lucotte, M.; Mergler, D. Daily mercury intake in fish-eating populations in the Brazilian Amazon. J. Expo. Sci. Environ. Epidemiol. 2008, 18, $76-87$. [CrossRef] [PubMed]

39. Fillion, M.; Passos, C.J.S.; Lemire, M.; Fournier, B.; Mertens, F.; Guimarães, J.R.D.; Mergler, D. Quality of life and health perceptions among fish-eating communities of the Brazilian amazon: An ecosystem approach to well-being. Ecohealth 2009, 6, 121-134. [CrossRef] [PubMed]

40. Grotto, D.; Valentini, J.; Fillion, M.; Passos, C.J.; Garcia, S.C.; Mergler, D.; Barbosa, F., Jr. Mercury exposure and oxidative stress in communities of the Brazilian Amazon. Sci. Total Environ. 2010, 408, 806-811. [CrossRef] [PubMed]

41. De Bortoli, M.C. Assessment of Thyroid Hormone (T3) Levels and Selenium Status of Women Living in Mercury Exposure Area; Universidade de São Paulo: de São Paulo, Brazil, 2009.

42. Barcelos, G.R.M.; Marco, K.C.; Grotto, D.; Valentino, J.; Garcia, S.C.; Braga, G.U.L.; Barbosa, F., Jr. Evaluation of Glutathione S-transferase GSTM1 and GSTT1 Polymorphisms and Methylmercury Metabolism in an Exposed Amazon Population. J. Toxicol. Environ. Health Part A 2012, 75, 960-970. [CrossRef] [PubMed]

43. Dutra, M.D.S.; Jesus, I.M.; Santos, E.C.O.; Lima, M.O.; Medeiros, R.L.F.; Cavadas, M.; Luiz, R.R.; Câmara, V.M. Longitudinal assessment of mercury exposure in schoolchildren in an urban area of the Brazilian Amazon. Cad. Saude Publica 2012, 28, 1539-1545. [CrossRef] [PubMed]

44. Farias, L.A.; Fávaro, D.I.T.; Pessoa, A.; Aguiar, J.P.L.; Yuyama, L.K.O. Mercury and methylmercury concentration assessment in children's hair from Manaus, Amazonas State, Brazil. Acta Amaz. 2012, 42, 279-286. [CrossRef]

45. Khoury, E.D.T.; Souza, G.S.; Silveira, L.C.L.; Araújo, A.A.; Pinheiro, M.C.N. Neurological manifestations in riverine populations from areas exposed to mercury in the Brazilian Amazon. Cad. Saude Publica 2013, 29, 2307-2318. [CrossRef] [PubMed]

46. Rocha, A.V.; Cardoso, B.R.; Cominetti, C.; Bueno, R.B.; Bortoli, M.C.; Farias, L.A.; Favaro, D.I.T.; Camargo, L.M.A.; Cozzolino, S.M.F. Selenium status and hair mercury levels in riverine children from Rondônia, Amazonia. Nutrition 2014, 30, 1318-1323. [CrossRef] [PubMed]

47. Faial, K.; Deus, R.; Deus, S.; Neves, R.; Jesus, I.; Santos, E.; Alvez, C.N.; Brasil, D. Mercury levels assessment in hair of riverside inhabitants of the Tapajós River, Pará State, Amazon, Brazil: Fish consumption as a possible route of exposure. J. Trace Elem. Med. Biol. 2015, 30, 66-76. [CrossRef] [PubMed]

48. Castilhos, Z.; Rodrigues-Filho, S.; Cesar, R.; Rodrigues, A.P.; Villas-Boas, R.; Jesus, I.; Lima, M.; Faial, K.; Miranda, A.; Brabo, E.; et al. Human exposure and risk assessment associated with mercury contamination in artisanal gold mining areas in the Brazilian Amazon. Environ. Sci. Pollut. Res. 2015, 22, 11255-11264. [CrossRef] [PubMed]

49. Hoshino, A.; Pacheco-Ferreira, H.; Sanches, S.G.G.; Carvallo, R.; Cardoso, N.; Perez, M.; Câmara, V.M. Mercury exposure in a riverside Amazon population, Brazil: A study of the ototoxicity of methylmercury. Int. Arch. Otorhinolaryngol. 2015, 19, 135-140. [CrossRef] [PubMed] 
50. Rocha, A.V.; Cardoso, B.R.; Zavarize, B.; Almondes, K.; Bordon, I.; Hare, D.J.; Favaro, D.I.T.; Cozzolino, S.M.F. GPX1 Pro198Leu polymorphism and GSTM1 deletion do not affect selenium and mercury status in mildly exposed Amazonian women in an urban population. Sci. Total Environ. 2016, 571, 801-808. [CrossRef] [PubMed]

51. De Carvalho, L.V.B. Avaliação dos Níveis de Estresse Oxidativo Induzido por Exposição ao Mercúrio em População Ribeirinha Infantojuvenil do Rio Madeira (RO); Escola Nacional de Saúde Pública Sergio Arouca: Rio de Janeiro, Brazil, 2016.

52. Barbosa, A.C.; Garcia, A.M.; Souza, J.R. Mercury contamination in hair of riverine populations of Apiacas reserve in the Brazilian Amazon. Water Air Soil Pollut. 1997, 97, 1-8. [CrossRef]

53. Soares de Campos, M.; Sarkis, J.E.S.; Müller, R.C.S.; Brabo, E.S.; Santos, E.C.O. Correlation between mercury and selenium concentrations in Indian hair from Rondônia State, Amazon region, Brazil. Sci. Total Environ. 2002, 287, 155-161. [CrossRef]

54. Pinheiro, M.C.N.; Muller, R.C.S.; Sarkis, J.E.; Vieira, J.L.F.; Oikawa, T.; Gomes, M.S.V.; Guimarães, G.A.; do Nascimento, J.L.M.; Silveira, L.C.L. Mercury and selenium concentrations in hair samples of women in fertile age from Amazon riverside communities. Sci. Total Environ. 2005, 349, 284-288. [CrossRef] [PubMed]

55. Lebel, J.; Mergler, D.; Lucotte, M.; Amorin, M.; Dolbec, J.; Miranda, D.; Aranttes, G.; Rheaut, R.; Pichet, P. Evidence of early nervous system dysfunction in Amazonian populations exposed to low-levels of methylmercury. Neurotoxicology 1996, 17, 157-167. [PubMed]

56. Lebel, J.; Mergler, D.; Branches, F.; Lucotte, M.; Amorim, M.; Larribe, F.; Dolbec, J. Neurotoxic effects of low-level methylmercury contamination in the Amazonian Basin. Environ. Res. 1998, 79, 20-32. [CrossRef] [PubMed]

57. Rodrigues, A.R.; Souza, C.R.B.; Braga, A.M.; Rodrigues, P.S.S.; Silveira, A.T.; Damim, E.T.B.; Côrtes, M.I.T.; Castro, A.J.O.; Mello, G.A.; Vieira, J.L.F. Mercury toxicity in the Amazon: Contrast sensitivity and color discrimination of subjects exposed to mercury. Braz. J. Med. Biol. Res. 2007, 40, 415-424. [CrossRef] [PubMed]

58. Santos, E.C.O.; Câmara, V.M.; Jesus, I.M.; Brabo, E.S.; Loureiro, E.C.B.; Mascarenhas, A.F.S.; Fayal, K.F.; Sá Filho, G.C.; Sagica, F.E.S.; Lima, M.O.; et al. A contribution to the establishment of reference values for total mercury levels in hair and fish in Amazonia. Environ. Res. 2002, 90, 6-11. [CrossRef] [PubMed]

59. Santos, E.C.O.; Jesus, I.M.; Brabo, E.S.; Fayal, K.F.; Sá Filho, G.C.; Lima, M.O.; Miranda, A.M.M.; Mascarenhas, A.S.; de Sá, L.L.C.; Silva, A.P.; et al. Exposure to mercury and arsenic in Amazon States: A summary of studies by the Evandro Chagas Institute/FUNASA. Rev. Bras. Epidemiol. 2003, 6, 171-185. [CrossRef]

60. Marques, R.C.; Dórea, J.G.; Bastos, W.R.; Rebelo, M.F.; Fonseca, M.F.; Malm, O. Maternal mercury exposure and neuro-motor development in breastfed infants from Porto Velho (Amazon), Brazil. Int. J. Hyg. Environ. Health 2007, 210, 51-60. [CrossRef] [PubMed]

61. Dorea, J.; Barbosa, A.C.; Ferrari, I.; de Souza, J.R. Mercury in hair and in fish consumed by Riparian women of the Rio Negro, Amazon, Brazil. Int. J. Environ. Health Res. 2003, 13, 239-248. [CrossRef] [PubMed]

62. Larson, H.J. The Minamata convention on mercury: Risk in perspective. Lancet 2014, 383, 198-199. [CrossRef]

63. Telmer, K.; Costa, M.; Simões Angélica, R.; Araujo, E.S.; Maurice, Y. The source and fate of sediment and mercury in the Tapajós River, Pará, Brazilian Amazon: Ground- and space-based evidence. J. Environ. Manag. 2006, 81, 101-113. [CrossRef] [PubMed]

64. WHO/UNEP DTIE Chemicals Branch. Guidance for Identifying Populations at Risk from Mercury Exposure; World Health Organization: Geneva, Switzerland, 2008; p. 167.

(C) 2018 by the authors. Licensee MDPI, Basel, Switzerland. This article is an open access article distributed under the terms and conditions of the Creative Commons Attribution (CC BY) license (http://creativecommons.org/licenses/by/4.0/). 